\title{
El Fondo de Desarrollo de Vuelos de Canarias: incentivos públicos para nuevas rutas aéreas en un destino turístico maduro
}

\author{
David Ramos-Pérez (a) \\ (a) Departamento de Geografía, Universidad de Salamanca, a13004@usal.es
}

\begin{abstract}
Resumen
La generalización de los incentivos públicos para la creación de nuevas conexiones aéreas es una de las consecuencias de la liberalización del mercado comunitario de transporte aéreo. Sin embargo, la literatura académica ha prestado una atención limitada a esta práctica, observándose una escasez de estudios de caso que permitan un conocimiento más detallado de la misma. La comunicación contribuye a solventar esa laguna, presentando los resultados de una investigación sobre el Fondo de Desarrollo de Vuelos vigente desde 2014 en Canarias, un destino turístico maduro que cuenta con una elevada conectividad aérea. A partir del análisis de fuentes documentales y estadísticas se determinan las causas que motivaron la puesta en marcha estos incentivos, la inversión realizada, las características de las rutas apoyadas y su contribución al cumplimiento de los objetivos perseguidos. Aunque el Fondo ha contribuido escasamente a la mejora de la conectividad aérea del archipiélago, puede hablarse de un impacto diferencial en función de la isla considerada.
\end{abstract}

Palabras clave: Transporte aéreo, liberalización, incentivos, rutas aéreas, Canarias.

\section{INTRODUCCIÓN}

Los incentivos económicos de carácter público otorgados a las compañías aéreas para la apertura de nuevas rutas son la consecuencia de dos procesos paralelos que se han desarrollado durante los últimos treinta años: la liberalización del transporte aéreo y la creciente competencia entre territorios que caracteriza la lógica espacial del capitalismo de acumulación flexible. El primero ha restringido notablemente la capacidad de los gobiernos para determinar no solo las conexiones aéreas, sino la capacidad ofrecida y sus tarifas, decisiones ahora en mano de las aerolíneas. Asimismo, al ir acompañada de la progresiva privatización, tanto de las compañías aéreas como de la gestión de los aeropuertos, ha aparecido una intensa competencia entre estos últimos para captar nuevas operaciones que incrementen su tráfico, con el objetivo último de ensanchar los márgenes de beneficio, tal y como exigen sus accionistas de referencia. El segundo, la competencia entre territorios, mantiene una estrecha relación con la creciente financiarización de la economía en el contexto de la globalización y la restructuración económica que ha afectado a un amplio conjunto de ciudades y regiones de los espacios centrales de la economíamundo capitalista, fruto de la intensificación de la división internacional del trabajo y la consiguiente desindustrialización de esos lugares en beneficio de las semi-periferias emergentes.

En ese contexto, las ciudades y regiones han sido impulsadas a competir entre sí a escala mundial con objeto de captar una parte del capital internacional, extremadamente móvil y selectivo, como ha señalado Harvey (1989). De ahí que las administraciones públicas que gestionan ambas escalas utilicen sus competencias para movilizar las ventajas comparativas de sus territorios, fundamentalmente a través de economías de oferta complementarias de las ya practicadas a escala estatal (Jessop, 2008). En estos espacios, las estrategias de desarrollo urbano y regional han seguido dos orientaciones básicas: el fomento de las actividades productivas vinculadas a la economía del conocimiento y creativa y la atracción de consumidores externos, mediante la promoción de los usos turísticos y de una oferta de experiencias (Hall, 2009). En ambos casos, la continua mejora de la conectividad aérea se ha considerado una ventaja comparativa clave a la hora de atraer capitales y turistas. En lo que respecta al turismo, como estos espacios centrales ofrecen productos similares basados en los atractivos de lo que genéricamente podríamos denominar la cultura urbana, su intercambiabilidad como destinos es alta, por lo que las características de la oferta área, como diversidad de rutas y la presencia de compañías que ofrezcan tarifas reduci- 
das, puede ser fundamental a la hora de decantar a los potenciales turistas. De ahí la trascendencia de los incentivos para la apertura de nuevas rutas, pues neutralizan parte del riesgo que para una aerolínea supone es decisión. Si la ruta no se consolida a corto plazo, el operador abandona la explotación del servicio, tras haber minimizado sus costes fijos gracias a esos fondos públicos. Por ello esta práctica, como las restantes que integran el marketing aeroportuario (Halpern y Graham, 2013), constituye un ejemplo meridiano de las economías de oferta a las que alude Jessop, idénticas en su concepción a las transferencias a la administración de ciertos costes en capital fijo que se observan de forma recurrente en todo tipo de inversiones que se materializan en estos espacios centrales del capitalismo.

En el caso de los destinos maduros de sol y playa, la dependencia de la conectividad aérea se remonta, al menos en la cuenca Mediterránea a la década de los años 60 del siglo XX. Asimismo, el producto sol y playa tampoco ha quedado al margen de la intensificación de la división espacial del trabajo, con la creciente concurrencia de nuevos destinos situados en espacios semi-periféricos y periféricos, que toma cuerpo en los años 70 y 80 (Gormsen, 1981) y se acentúa a lo largo del siglo XXI (Navarro Jurado et al., 2015). A ello se le une un cierto cambio en los patrones de consumo de los potenciales turistas, entre los que gana peso el viaje individual frente al tradicional paquete, se observa una mayor demanda de flexibilidad en las fechas de viaje frente a los calendarios fijos de turoperadores y compañías chárter y se constata la tendencia minimizar los costes del viaje aéreo, en consonancia con las pautas derivadas de la difusión de las compañías de bajo coste. En ese sentido, en los destinos emergentes de países menos desarrollados, las compañías aéreas, especialmente de bajo coste, han sido generosamente subsidiadas para asegurar una conectividad mínima que permitiese el arranque de los mismos, como muestran los ejemplos de Marruecos, Egipto, Israel o Tailandia, por citar solo algunos ejemplos de una amplia lista. De ahí que estos destinos hayan terminado diseñando incentivos para intentar asegurar una mayor presencia de operadores de bajo coste y/o una diversificación de los mercados emisores, como se observa en los casos de Malta (Graham y Dennis, 2010) y Faro (Ramos-Pérez e Izquierdo-Misiego, 2015).

La comunicación inserta el análisis del Fondo de Desarrollo de Vuelos vigente en Canarias desde 2014 en ese contexto, presentando a partir de diferentes fuentes documentales sus antecedentes y los factores que justificaron su diseño actual, así como sus principales características. Posteriormente se analizan las 103 convocatorias de rutas abiertas entre julio de 2014 y abril de 2018, para lo que se ha elaborado una base de datos que incorpora la información disponible en la web del Fondo de Desarrollo de Vuelos. Ello permite conocer la distribución de las rutas entre los diferentes aeropuertos de las islas, la situación administrativa de las convocatorias y su contribución a la diversificación de la oferta programada en dichos aeropuertos. También se analiza la inversión realizada y se relaciona con el retorno esperado en función de las llegadas de turistas previstos. Se concluye apuntando la necesidad de comprobar el grado de cumplimento del Fondo de Desarrollo de Rutas una vez termine su vigencia, dada la necesidad de verificar el mantenimiento de las conexiones aérea una vez desaparecidos los incentivos.

\section{ORIGEN DEL FONDO DE DESARROLLO DE VUELOS}

El Fondo de Desarrollo de Vuelos de Canarias es la materialización del Programa de Conectividad Aérea para el archipiélago previsto en el Acuerdo por la Competitividad y la Calidad del Turismo en Canarias 2008-2020, firmado en Fuerteventura en octubre de 2007. En dicho documento, las administraciones públicas canarias y las principales organizaciones empresariales representativas del sector turístico canario acordaron la necesidad de poner en marcha de forma inmediata un conjunto de medidas que permitieran fortalecer las islas como un destino turístico de referencia en los mercados emisores europeos. El contexto de creciente competencia con nuevos destinos especializados en el producto de sol y playa y la amplia difusión de nuevos patrones de consumo entre los potenciales turistas estaba dando lugar a cambios estructurales a los que se quería hacer frente. De ahí que las cuestiones relacionadas con la conectividad aérea fueran consideradas un pilar básico del Plan Estratégico del Destino Canarias, junto con la mejora del conocimiento existente sobre los mercados emisores y la reformulación de la promoción del archipiélago el exterior.

En todo caso, antes de este acuerdo, el Gobierno de Canarias ya había demostrado cierto interés por la conectividad aérea, al poner en marcha a finales de 2006 un Fondo de Desarrollo de Vuelos, dotado de un presupuesto inicial de 1 millón de euros, que estaba previsto incrementar en medio millón cada año. El Fondo se diseñó como un programa de incentivos abierto a cualquier compañía que quisiera incrementar las frecuencias operadas en una ruta existente o poner en marcha una nueva conexión, para lo que se ofrecían 1 y 2 euros respectivamen- 
te por asiento de salida ofrecido. En su corta andadura 7 compañías que respondían a modelos de negocio sumamente diferentes se beneficiaron de estos incentivos. Aunque el Fondo probablemente cumplía con la legislación comunitaria en materia de ayudas de estado en vigor desde 2005, a diferencia de lo que ocurría con las ayudas que otorgaban otros gobiernos locales y regionales del país (Ramos-Pérez, 2016), no era resultado de ningún análisis estratégico previo ni se conocen sus objetivos, más allá de los genéricos de "captar todo tipo de tráfico aéreo" o el "aumento de las conexiones para favorecer un abaratamiento de tarifas" que señalaba el consejero de turismo en su presentación ${ }^{1}$. Todo indica que la acelerada difusión de las compañías de bajo coste en el mercado intracomunitario que se observaba en aquellos años (Dobruszkes, 2006) y la relativa ausencia de las mismas en las rutas que conectaban las islas con sus mercados emisores, llevaron a tomar esa medida en un momento en que eran ampliamente conocidas las exigencias de algunas compañías de bajo coste, especialmente Ryanair, para operar en algunos destinos.

El nuevo Fondo, como hemos anticipado, es el resultado de una profunda reflexión sobre los cambios estructurales que venían afectando al turismo desde hacía al menos una década. De ahí que sólo sea aprobado tras la elaboración de un Plan Estratégico de Conectividad Aérea de las Islas Canarias (PECAIC), (Consejería de Turismo, 2009), que radiografiaba la situación de las conexiones aérea en el archipiélago en aquel momento, analizaba los cambios que se observaban a escala europea en los distintos modelos de negocio de las compañías aérea, señalaba las nuevas demandas de los turistas en cuanto a movilidad aérea e incidía en la debilidad que la excesiva dependencia de los turoperadores suponía para el destino, incluyendo las dificultades de acceso a la compra directa de billetes para ese nuevo perfil de turistas.

En ese contexto de cambios y transformaciones, el Plan apuntaba el desinterés que comenzaban a manifestar los turoperadores por un destino como Canarias, donde los márgenes de beneficio eran claramente inferiores a los que era posible obtener en destinos que ofrecían un producto similar, como Turquía y Egipto (Consejería de Turismo, 2009). De ahí que el riesgo de un creciente desvío de turistas hacia los mismos pudiese reducir la demanda hacia el archipiélago, especialmente durante una etapa en la que la crisis económica y financiera iba a reducir el poder adquisitivo de los potenciales turistas. Si a ello se suma la existencia de incentivos económicos que los destinos turísticos emergentes estaban otorgando a las compañías aéreas y turoperadores para abaratar sus costes operativos, se concluía que Canarias estaba experimentando una pérdida de competitividad que era necesario atenuar. Por ello proponía un conjunto de actuaciones destinadas a desarrollar una verdadera política de conectividad aérea, que permitiese atenuar esa pérdida de competitividad en lo que respecta a las conexiones aéreas. Aunque la propuesta era ambiciosa, incluyendo la creación de un departamento específico sobre conectividad aérea en el seno de la Consejería de Turismo, debido a la notable especialización que requiere la cuestión, únicamente el Fondo de Desarrollo de Vuelos ha terminado adoptándose.

Por tanto, a diferencia de lo que ocurría en otros territorios, en los que los programas de desarrollo de rutas aéreas se han diseñado para abordar los déficits de conectividad aérea detectados, como es el caso del Scottish Route Development Fund (Smyth et al., 2012), en Canarias no existía una situación de reducida conectividad aérea. En ese sentido, el Fondo de Desarrollo de Vuelos es sobre todo un intento de hacer frente a la creciente competencia de destinos turísticos que presentan un menor desarrollo económico y cuentan con una normativa que permite los subsidios directos a las aerolíneas y los turoperadores, lo que incrementa sus márgenes de beneficio. Es decir, el Fondo es una herramienta para paliar las consecuencias de una competencia desigual entre destinos fruto de la globalización del turismo, facilitando los márgenes de beneficio de las aerolíneas y turoperadores interesados en conectar las islas con nuevos mercados emisores.

La cuestión normativa no es un aspecto menor, pues la legislación comunitaria prohíbe las ayudas de estado en sectores liberalizados como el transporte aéreo, aun cuando existen determinadas excepciones al respecto. Las Directrices aprobadas por la Comisión Europea en el año 2005 (DOUE, 2005) venían a clarificar en qué circunstancias eran compatibles con el mercado interior, al objeto de poner fin a los subsidios que mediante diversas fórmulas se otorgaban de forma opaca en múltiples aeropuertos de la Unión Europea. La apertura de varias investigaciones al respecto por parte de la Comisión Europea propició que el Gobierno de Canarias renunciase al antiguo Fondo de Desarrollo de Vuelos y optase por un nuevo planteamiento, que cumpliese con dichas Directrices y tuviese el visto bueno de la Comisión.

\footnotetext{
${ }^{1}$ El Gobierno de Canarias destina un millón de euros a la creación de un Fondo de Desarrollo de Vuelos. Nota de prensa del Gobierno de Canarias (27/09/2006). Disponible en http://www.gobcan.es/noticias/tcd/Turismo/36566/gobierno-canarias-destina-millon-euros-creacion-fondo-desarrollo -vuelos
} 


\section{CARACTERÍSTICAS DEL FONDO DE DESARROLLO DE VUELOS}

En julio de 2013, cuatro años después de elaborado el PECAIC, el Gobierno de Canarias solicita formalmente a la Comisión Europea la autorización para un Programa de desarrollo de vuelos en el territorio de la región ultraperiférica de las Islas Canarias para 2013-2017, dotado con un presupuesto de 10 millones de euros, al objeto de incentivar la puesta en marcha de nuevas rutas aéreas con origen o destino en los aeropuertos del archipiélago (CE, 2014). Estos incentivos se otorgarían mediante convocatorias públicas, tendrían la condición de subvenciones directas a las compañías aéreas con el objeto de financiar hasta el 50\% de los costes asociados al pago de las tasas aeroportuarias y podrían prolongarse durante un periodo máximo de tres años. Al fijar como fecha límite para la apertura de nuevos destinos el 31 de diciembre de 2017, los pagos finalizarían en el año 2020.

Se cumplía así con todos los criterios establecidos en la nueva versión de las Directrices Comunitarias sobre ayudas estatales a aeropuertos y compañías aéreas (DOUE, 2014), cuya aprobación coincidió en el tiempo con la solicitud de autorización del Gobierno de Canarias, pero cuyo contenido era conocido gracias al periodo de consultas abierto por la Comisión antes de su aprobación. Además, el especial tratamiento otorgado en las Directrices a las regiones ultraperiféricas ${ }^{2}$, permitió que todos los aeropuertos del archipiélago pudiesen beneficiarse de estos incentivos, cuando generalmente solo se autorizan para aeropuertos con un tráfico inferior a los tres millones de pasajeros, circunstancia que en Canarias solo cumplirían La Palma, El Hierro y La Gomera.

Al objeto de evitar que estas ayudas pudieran dar lugar a una situación de competencia desleal, los tipos de rutas que podían beneficiarse de las mismas también quedan claramente definidos a través de una serie de criterios acumulativos (CE, 2014, p.6):

- Rutas que no hayan sido operadas por una compañía aérea que ofrezca servicios de transporte de pasajeros de pago en los 12 meses anteriores a la fecha prevista para el inicio de la explotación de los nuevos servicios.

- Rutas en relación con las cuales, con anterioridad a la publicación de la convocatoria de propuestas, no haya habido ningún anuncio por parte de alguna compañía aérea de que se hubiera previsto su explotación durante el período de 12 meses siguiente a la publicación.

- Rutas a destinos que no es probable que den lugar a una transferencia de pasajeros de una ruta existente en otro aeropuerto canario.

- Rutas que conecten exclusivamente otro aeropuerto situado fuera de la región con cualquiera de los aeropuertos canarios.

Asimismo, también se señala que no se otorgarán ayudas cuando se trata de un incremento de frecuencias de una ruta ya explotada, lo que evita también posibles denuncias por competencia desleal en unas conexiones de marcado carácter turístico cuya oferta de vuelos se limita, en la mayoría de las rutas, a una o dos operaciones semanales.

El Programa tenía el objetivo de incentivar un máximo de 50 rutas, cifra que a mediados de 2017 se comprobó que resultaría inalcanzable. Dado que el presupuesto estaba lejos de agotarse, El Gobierno de Canarias solicitó a la Comisión Europea en agosto de 2017 la extensión del programa durante otros cuatro años manteniendo el presupuesto original. De esta manera se podrán conceder incentivos hasta el 31 de diciembre de 2021 y las rutas podrán operar hasta final de 2024 (CE, 2017).

\section{LAS CONVOCATORIAS DE RUTAS DEL FONDO DE DESARROLLO DE VUELOS}

Entre julio de 2014 y abril de 2018, la empresa pública Promotur, responsable del estudio y promoción del archipiélago como destino turístico, ha abierto 103 convocatorias para otorgar incentivos económicos a cargo del Fondo de Desarrollo de Vuelos. Esas 103 convocatorias se corresponden con 81 rutas aéreas, porque en 16 rutas los incentivos se han convocado en dos ocasiones y en otras 3 se ha llegado a las tres convocatorias con la finalidad de que la adjudicación no quedase desierta.

Las convocatorias se han abierto en ocho rondas diferentes que, a partir de 2017, presentan carácter anual.

\footnotetext{
${ }^{2}$ Las Directrices introducen un tratamiento específico en virtud de su enclavamiento para las denominadas regiones remotas, categoría en la que incluyen a las regiones ultraperiféricas, Malta, Chipre, Ceuta, Melilla, islas que forman parte del territorio de un Estado miembro y regiones poco pobladas.
} 
En un primer momento los incentivos se ofrecían para explotar una frecuencia semanal, bien durante la temporada de invierno (21 semanas) bien durante el verano (31 semanas), con una oferta mínima semanal de 180 asientos de llegada durante al menos dos años. Este esquema ha variado ligeramente en las dos últimas rondas de convocatorias, pues ahora no se determina previamente la temporada de operación, aspecto que se deja a criterio de la compañía, existiendo también la posibilidad de proponer una operación permanente durante todo el año.

Hasta el momento, las rutas para las que se convocan incentivos se distribuyen casi exclusivamente entre las islas de Gran Canaria (LPA), Tenerife (TCI), Lanzarote (ACE), La Palma (SPC) y Fuerteventura (FUE), aquellas que cuentan con aeropuertos capaces de recibir reactores de tamaño medio (figura 1). Las dos convocatorias que afectan a la isla de La Gomera (QGZ), que preveían rutas directas con Londres y Frankfurt en pequeños reactores de hasta 100 plazas, constituyen la excepción, y parecen responder más a las presiones políticas del Cabildo de La Gomera para no dejar su aeropuerto al margen del Fondo de Desarrollo de Vuelos que a la posibilidad real de que estos aviones puedan operar en una pista de 1.500 metros de longitud. En caso de El Hierro (VDE), donde la pista aún es de menores dimensiones, no se han propuesto incentivos para ninguna ruta.

De las cinco islas mencionadas, Fuerteventura es la que se ha visto afectada en menor medida por las convocatorias de rutas, mientras Gran Canaria y Tenerife se sitúan en el extremo opuesto, ocupando Lanzarote y La Palma una situación intermedia. La oferta de asientos asociada a las convocatorias de rutas replica esta situación, ya que rige el planteamiento anteriormente esbozado de una oferta mínima semanal de una frecuencia por ruta.

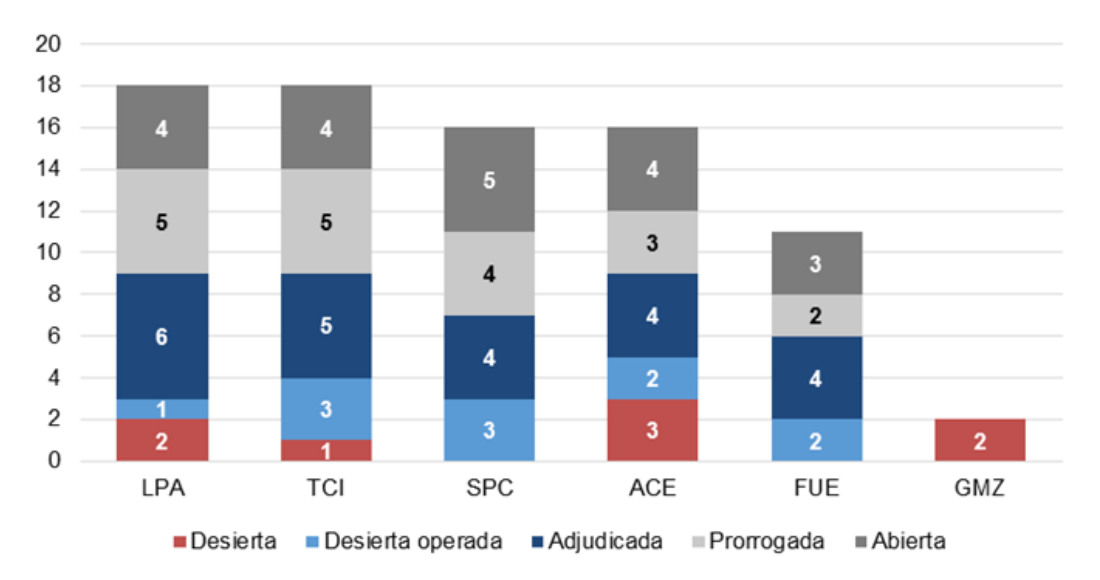

Figura 1 - $\mathrm{N}^{\circ}$ de rutas convocadas por aeropuerto y situación del concurso. Fuente: Elaboración propia a partir de los datos disponibles en http://turismodeislascanarias.com/ es/fondo-de-desarrollo-de-vuelos

Especialmente relevante es el hecho de que únicamente 23 de las convocatorias realizadas hasta el momento han dado lugar a la puesta en marcha de una nueva ruta, lo que supone una efectividad del $28,4 \%$ (figuras 1 y 2). Si no consideramos las últimas 20 convocatorias, que fueron oficialmente presentadas el 20 de abril de 2008 , esa efectividad se eleva al $37,7 \%$. Sumando las 11 convocatorias oficialmente declaradas desiertas pero que durante la temporada de invierno 2017-18 eran operadas por alguna aerolínea, las propuestas exitosas alcanzarían el $56 \%$ de los casos. Es decir, prácticamente la mitad de las nuevas rutas planteadas por Promotur no resultan atractivas para ninguna compañía.

Los datos disponibles también indican que en Fuerteventura y La Palma las posibilidades de que una ruta quede desierta son menores. Además, cuando ello ha ocurrido, al cabo del tiempo una compañía ha terminado ofertando dichas rutas sin recurrir a los incentivos, por lo que el objetivo de establecer esa conexión ha terminado consiguiéndose. Sin embargo, conviene señalar que, a partir de la convocatoria de 2017, Promotur ha decidido no declarar desiertas las adjudicaciones de las rutas, manteniendo una prórroga permanente de las mismas cuando se cumple la fecha límite para presentar solicitudes sin haber recibido propuesta alguna. Ello introduce un sesgo notable a la hora de evaluar los resultados del Fondo de Desarrollo de Vuelos, por lo que solo tras la finalización del mismo en 2021 será posible conocer la totalidad de rutas por las que no se ha interesado ninguna compañía.

Las 81 rutas aéreas incluidas en las convocatorias involucran a 57 aeropuertos situados en 24 países diferentes. Destacan por el número de rutas que concentran Italia (12), Polonia y Francia (9), mercados emisores secundarios para el archipiélago que Promotur trata de consolidar para así reducir la dependencia frente al Reino 


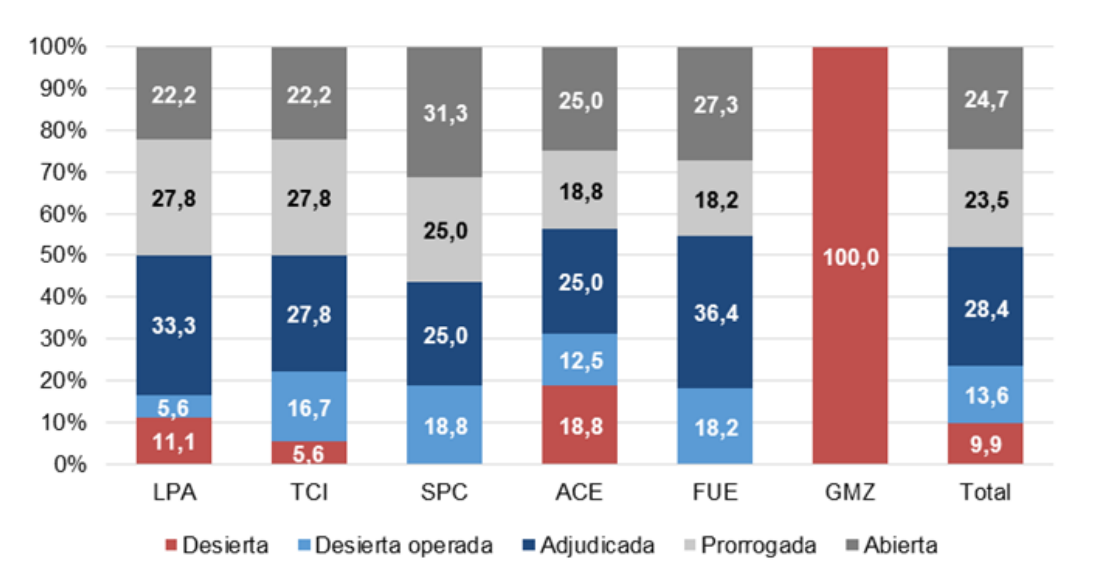

Figura 2 - Porcentaje de rutas convocadas por aeropuerto y situación del concurso. Fuente: Elaboración propia a partir de los datos disponibles en http:// turismodeislascanarias.com/es/fondo-de-desarrollo-de-vuelos

Unido, Alemania y los países escandinavos. A ellos se unen otros mercados emisores emergentes del este de Europa, como Eslovaquia y Hungría, así como otros maduros, caso de Suiza y Austria, en los que había descendido el flujo de turistas emitido hacia el archipiélago. En todo caso, existen situaciones particulares de algunas islas, como La Palma, donde el Reino Unido, Alemania, Dinamarca y Suecia concentran el grueso de las convocatorias de rutas. El menor desarrollo del turismo en la misma y la apuesta por consolidar o incluso abrir esos mercados tradicionales en el resto del archipiélago explican esta especificidad.

Si nos centramos en las 23 rutas operadas gracias a los incentivos, éstas solo involucran a 16 aeropuertos de 10 países, superando en este caso las conexiones con Francia y Hungría a las existentes con Polonia e Italia. Al igual que ocurría con el total de rutas convocadas, los enlaces con esos países son predominantes salvo en el caso de La Palma, donde las rutas con Dinamarca y Suecia son las únicas que por el momento reciben incentivos.

En lo tocante al modelo de negocio de las compañías beneficiadas por los incentivos, las aerolíneas de bajo coste operan 18 de las 23 rutas, destacando la húngara Wizz Air (6 rutas), la española Vueling (5 rutas) o la irlandesa Ryanair (3 rutas). Cabe destacar también la presencia de la antigua compañía chárter escandinava Primera Air, que recibe ayudas por las 4 rutas que conectan La Palma con las principales ciudades suecas y danesas.

Cuadro 1 - Rutas que han recibido incentivos del FDV según el país de origen y el aeropuerto canario de destino. Fuente: Análisis propio a partir de los datos disponibles en http:// turismodeislascanarias.com/es/fondo-de-desarrollo-de-vuelos

\begin{tabular}{|c|c|c|c|c|c|c|}
\hline País & ACE & FUE & LPA & SPC & $\mathrm{TCl}$ & TOTAL \\
\hline Francia & 1 & 2 & 1 & - & 1 & 5 \\
\hline Hungría & 1 & 1 & 1 & - & 1 & 4 \\
\hline Polonia & 1 & - & 1 & - & 1 & 3 \\
\hline Italia & 1 & - & 2 & - & - & 3 \\
\hline Suecia & - & - & - & 2 & - & 2 \\
\hline Dinamarca & - & - & - & 2 & - & 2 \\
\hline Finlandia & - & - & 1 & - & - & 1 \\
\hline Rumanía & - & - & - & - & 1 & 1 \\
\hline República Checa & - & 1 & - & - & - & 1 \\
\hline Portugal & - & - & - & - & 1 & 1 \\
\hline TOTAL & 4 & 4 & 6 & 4 & 5 & 23 \\
\hline
\end{tabular}

\section{EL IMPACTO DEL FONDO DE DESARROLLO DE VUELOS EN LOS AEROPUERTOS CANARIOS}

La oferta anual asociada a las rutas propuestas en las distintas convocatorias se sitúa por debajo de los 700.000 asientos de llegada, lo que, en 2016, último año para el que existen datos oficiales, suponía un 4,3\% de la oferta total de asientos internacionales en los aeropuertos canarios. Salvo en el caso del aeropuerto de La Pal- 
ma, donde el impacto potencial de estas rutas puede ser significativo, ya que la oferta propuesta supone el 55\% de la existente, en los otros aeropuertos los potenciales efectos del Fondo serán mucho más limitados. En todo caso, conviene no minimizar el hecho de que estas rutas pueden estabilizar una conexión con mercados emisores con los que solo existía vinculación a través de operaciones chárter.

En lo que respecta a las rutas puestas en marcha, esa oferta anual se reduce a los 189.000 asientos, apenas un $1,2 \%$ de la oferta total internacional del archipiélago. Al igual que con la oferta convocada, este valor medio encubre la existencia de una notable diferencia entre La Palma y el resto de las islas, siendo el aeropuerto en el que el Fondo de Desarrollo de Vuelos ha tenido una clara repercusión en la evolución reciente de la oferta. Los 15.120 asientos de los vuelos que conectan durante las temporadas de invierno La Palma con Copenhague, Billund, Gotemburgo y Estocolmo supondrían el 7,2\% de la oferta con la que contó la isla en el año 2016. Pero su peso es aún mayor si tenemos en cuenta que se trata de rutas estacionales, por lo que si nos ceñimos a la última te 54 mporada de invierno 2017-18, esas 4 conexiones suponían el 9,1\% de la oferta programada.

Además, al estar asociada la compañía aérea beneficiaria de los incentivos (Primera Air) a uno de los principales turoperadores escandinavos (Primera Travel Group), el resultado ha sido la puesta en marcha también durante la temporada de invierno de una ruta semanal entre La Palma y Oslo, así como de una serie de operaciones con origen en diversos aeropuertos finlandeses, que suman una oferta adicional de 6.897 plazas. Si esas plazas las añadimos a la oferta que recibe incentivos, resulta que el $13,3 \%$ de la oferta de asientos de llegada programada en

Cuadro 2 - Peso de la oferta de asientos de las rutas que reciben incentivos en la programación de la temporada de invierno 2017 18. Fuente: Análisis propio a partir de los datos disponibles en http://turismodeislascanarias.com/es/fondo-de-desarrollo-devuelos y de la oferta de asientos de la temporada de invierno 2017-2018 publicada por AENA en http://www.aena.es/ DescargaNotasPrensa/171031-can-temporada-invierno-20172018.pdf

\begin{tabular}{|c|c|c|c|}
\hline \multirow[b]{2}{*}{ Aeropuerto } & \multicolumn{2}{|c|}{ Oferta de asientos de llegada } & \multirow[b]{2}{*}{$\%$} \\
\hline & $\begin{array}{c}\text { En rutas } \\
\text { internacionales }\end{array}$ & $\begin{array}{l}\text { En rutas con } \\
\text { incentivos }\left({ }^{*}\right)\end{array}$ & \\
\hline LPA & 2.300 .000 & 18.900 & $0,8 \%$ \\
\hline $\mathrm{TCl}$ & 2.563 .500 & 18.900 & $0,7 \%$ \\
\hline ACE & 1.250 .000 & 15.120 & $1,2 \%$ \\
\hline FUE & 1.150 .000 & 15.120 & $1,3 \%$ \\
\hline SPC & 166.000 & 15.120 & $9,1 \%$ \\
\hline TOTAL & 7.429 .500 & 83.160 & $1,1 \%$ \\
\hline
\end{tabular}

NOTA: $\left(^{*}\right)$ Solo se consideran las rutas beneficiadas por los incentivos. La oferta está ajustada al $n^{\circ}$ de semanas (21) de la temporada de invierno. La Palma el pasado invierno puede relacionarse con el Fondo de Desarrollo de Vuelos.

\section{LA RELACIÓN COSTE-BENEFICIO DE LAS RUTAS QUE HAN RECIBIDO INCENTIVOS}

El Plan Estratégico de Conectividad Aérea de las Islas Canarias (Consejería de Turismo, 2009) estimaba el beneficio que cada nueva ruta operada supondría para el archipiélago a partir del gasto medio diario imputable a los nuevos turistas captados por la existencia de estas conexiones. En dicho cálculo no se introducían variables de tipo ambiental, que por ejemplo tuvieran en cuenta la contribución a las emisiones de efecto invernadero de estas nuevas rutas o el incremento de la presión antrópica sobre el medio asociada al aumento de los turistas. $Y$ tampoco sociales, vinculadas por ejemplo con el modelo de relaciones laborales dominante en las compañías seleccionadas.

Si no introducimos dichas variables en el balance, y seguimos el planteamiento del PECAIC, actualizando los valores de la estancia media del turista (9,17 días en 2017$)$ y del gasto medio $(140,18 €)$, considerando una ocupación del $80 \%$ en las rutas y una proporción del tráfico de llegada (inbound) del $95 \%$, se obtiene que las 23 rutas operadas gracias a los incentivos generarían generando anualmente volumen de negocio de 184,6 M€. Dado que el coste anual asociado a los incentivos se sitúa en 1,5 M€, el balance resulta en ese sentido claramente positivo. Es más, si solo tenemos en cuenta el gasto medio diario que los turistas efectúan directamente en Canarias $(39,03 €)$, el volumen de negocio generado por los mismos se situaría en los 51,4 M€, también muy por encima del coste de los incentivos, lo que asegura un elevado retorno de la inversión. 


\section{CONCLUSIONES}

EI PECAIC estableció en 2009 una serie de prioridades respecto a las rutas a las que deberían concederse incentivos, señalando la necesidad de intervención urgente en un conjunto de rutas de los tres principales mercados emisores (Península, Alemania y Reino Unido) en las que se habían detectado grandes caídas de tráfico. Posteriormente recomendaba centrarse en un conjunto de mercados donde se había detectado un mayor potencial de crecimiento, como Francia, Italia, Rusia, Polonia o la República Checa. El cambio en la coyuntura turística explica que los incentivos otorgados hasta el momento se hayan orientado hacia las rutas con los mercados emisores emergentes para el archipiélago, salvo en el caso de La Palma, donde la puesta en marcha de enlaces con los países nórdicos ha sido priorizada.

Sin embargo, el impacto del Fondo en la diversificación de los mercados emisores de turistas hacia el archipiélago resulta marginal, como demuestra el peso de la oferta de asientos asociada a los incentivos sobre la oferta total. Si a ello le unimos que el $46 \%$ de las rutas para las que se ofrecen incentivos no ha recibido propuesta alguna por parte de las aerolíneas, parece existir un cierto desajuste entre una estrategia de captación de rutas ambiciosa y la indiferencia de las compañías aéreas respecto a la mayoría de las mismas, al menos hasta el momento. Es más, el elevado peso que las rutas del Fondo tienen en La Palma refuerza la idea original de la Comisión Europea de que estos mecanismos tienen un mayor impacto en aquellos territorios con una limitada conectividad aérea, siendo mucho más discutible su utilidad cuando se dispone de un amplísimo abanico de conexiones, como ocurre en las restantes islas del archipiélago.

Por otro lado, en Canarias, a diferencia de lo ocurrido en los pequeños aeropuertos regionales del interior peninsular (Ramos-Pérez, 2016), el balance coste-beneficio de los incentivos resulta positivo, puesto que el tráfico que canalizan las rutas a las que se otorgan las ayudas es fundamentalmente de llegada (tráfico inbound). Sin embargo, un análisis detallado de la efectividad del Fondo de Desarrollo de Vuelos sólo será posible a medio plazo, cuando pueda verificarse en qué medida las rutas que se han beneficiado de los incentivos continúan operándose en ausencia de los mismos.

\section{BIBLIOGRAFIA}

CE (Comisión Europea) (2014). Ayudas de puesta en marcha para las compañías aéreas que operan desde las Islas Canarias (SA.37121, 2013/N). Disponible en http://ec.europa.eu/competition/elojade/isef/case_details.cfm? proc_code=3_SA_37121

CE (Comisión Europea) (2017). Ampliación de un régimen de ayudas de puesta en marcha para las compañías aéreas que operan desde las Islas Canarias (SA.48872, 2017/N). Disponible en http://ec.europa.eu/competition/elojade/isef/case_details.cfm? proc_code=3_SA_48872

CONSEJERÍA DE TURISMO (2009). Plan Estratégico de Conectividad Aérea de las Islas Canarias (PECAIC). Gobierno de Canarias y Es klappt Consulting Estratégico \& Calidad. Disponible en http://www.datosdelanzarote.com/itemDetalles.asp? idFamilia=31\&idltem $=4340$

DOBRUSZKES F. (2006), An analysis of European low-cost airlines and their networks, Journal of Transport Geography, 14(4), 249-264.

DOCE (Diario Oficial de las Comunidades Europeas) (2005). Directrices Comunitarias sobre la financiación de aeropuertos y las ayudas estatales de puesta en marcha destinadas a compañías aéreas que operen desde aeropuertos regionales (Diario $\mathrm{n}^{\circ} \mathrm{C} 312$ de 09/12/2005, pp. 1-14). Luxemburgo: Oficina de Publicaciones Oficiales de las Comunidades Europeas.

DOCE (Diario Oficial de las Comunidades Europeas) (2014). Directrices sobre ayudas estatales a aeropuertos y compañías aéreas (Diario n ${ }^{\circ}$ C99 de 04/04/2014). Luxemburgo: Oficina de Publicaciones Oficiales de las Comunidades Europeas.

GORMSEN, E. (1981). The spatio-temporal development of international tourism. Attempt at a centre-periphery model. In Union Geographique Internationale (Ed.), La Consommation d'espace par le tourisme et sa preservation (pp. 150-170). Aix-enProvence: Centre Hautes Etudes Touristiques.

GRAHAM, A. Y DENNIS, N. (2008) The impact of low cost airline operations to Malta. Journal of Air Transport Management, 16 (3), 127-136

HALL, C.-M. (2009). El turismo como ciencia social de la movilidad. Madrid: Síntesis.

HALPERN, N. Y GRAHAM, A. (2013). Airport Marketing. Londres: Routledge.

HARVEY, D. (1989). From Managerialism to Entrepreneurialism: The Transformation in Urban Governance in Late Capitalism. 
Geografiska Annaler Series B, 71(1), 3-17.

JESSOP, R. (2008). El futuro del Estado capitalista. Madrid: Los Libros de la Catarata.

NAVARRO JURADO, E.; THIEL ELLUL, D. Y ROMERO PADILLA, Y. (2015). Periferias del placer: cuando turismo se convierte en desarrollismo inmobiliario-turístico. Boletín de la Asociación de Geógrafos Españoles, n 67, 275-302.

RAMOS-PÉREZ, D. (2016). State aid to airlines in Spain: An assessment of regional and local government support from 1996 to 2014. Transport Policy, 49, 137-147.

RAMOS-PÉREZ, D. E IZQUIERDO-MISIEGO, J.I. (2015). Flying from Europe to the Algarve: The Geographical Impacts of the Growth of Low-Cost Carriers (1996-2013). Journal of Spatial and Organizational Dynamics, 3 (4), 275-295

SMYTH, A., CHRISTODOULOU, G., DENNIS, N., AL-AZZAWI, M. Y CAMPBELL, J. (2012). Is air transport a necessity for social inclusion and economic development? Journal of Air Transport Management, 22, 53-59. 